\title{
Interactive comment on "Adaptive stratified importance sampling: hybridization of extrapolation and importance sampling Monte Carlo methods for estimation of wind turbine extreme loads” by Peter Graf et al.
}

Peter Graf et al.

peter.graf@nrel.gov

Received and published: 11 October 2017

The comment was uploaded in the form of a supplement:

https://www.wind-energ-sci-discuss.net/wes-2017-30/wes-2017-30-AC1-

supplement.pdf 